Proceedings of the ASME 2016, $35^{\text {th }}$ International Conference on Ocean, Offshore and Arctic Engineering

\title{
OMAE2016-54796
}

\section{NUMERICAL SIMULATION OF A TIDAL TURBINE BASED HYDROFOIL WITH LEADING-EDGE TUBERCLES}

\author{
Weichao Shi \\ School of Marine Science and Technology \\ Newcastle University \\ Newcastle upon Tyne, NE1 7RU, UK
}

\author{
Mehmet Atlar \\ School of Marine Science and Technology \\ Newcastle University \\ Newcastle upon Tyne, NE1 7RU, UK
}

\author{
Kwangcheol Seo \\ Department of Naval Architecture and \\ Ocean Engineering \\ Mokpo National Maritime University, \\ South-Korea
}

\author{
Rosemary Norman \\ School of Marine Science and \\ Technology \\ Newcastle University \\ Newcastle upon Tyne, NE1 7RU, UK
}

\author{
Roslynna Rosli \\ School of Marine Science and \\ Technology \\ Newcastle University \\ Newcastle upon Tyne, NE1 7RU, UK
}

\section{ABSTRACT}

The tubercles along the leading edges of the humpback whale flippers can provide these large mammals with an exceptional maneuverability. This is due to the fact that the leading-edge tubercles have largely a 3D benefit for the finite hydrofoils, which can maintain the lift, reduce the drag and delay the stall angle. Newcastle University launched a series study to improve a tidal turbine's performance with the aid of this concept.

This paper presents a numerical simulation of the tested hydrofoil, which is representative of a tidal turbine blade, to investigate the flow around the foil and also to numerically model the experiment. This hydrofoil was designed based on an existing tidal turbine blade with the same chord length distribution but a constant pitch angle. The model tests have been conducted in the Emerson Cavitation Tunnel measuring the lift and drag. The results showed that the leading-edge tubercles can significantly improve the performance of the hydrofoil by improving the lift-to-drag ratio and delaying the stall.

By applying Shear Stress Transport (SST), Detached Eddy Simulation (DES) and Large Eddy Simulation (LES) via using the commercial CFD solver, Star-CCM+, the tested hydrofoil models were simulated and more detailed flow information has been achieved to complement the experiment. The numerical results show that the DES model is in close agreement with the experimental results. The flow separation pattern indicates the leading-edge tubercles can energize the flow around the hydrofoil to keep the flow more attached and also separate the flow into different channels through the tubercles.

\section{INTRODUCTION}

Humpback whale is a species of giant marine mammal which has the ability to complete athletic maneuvers in order to catch prey. They utilize their unusually long pectoral fins to perform tight turns to drive a school of fish into a small circular zone in order to swallow them effectively. However, their long fins are not streamlined at the leading edges having some tubercles which are round shaped protuberances [1,2]. Wind tunnel tests showed that placing leading-edge tubercles along a blade could improve performance, such as delaying stall and providing higher lift-to-drag ratio [3-8]. Based on these results a number of numerical and experimental research studies have been conducted in order to understand the tubercle concept $[3,5$, 6, 9-13]. The 3D foil applications that refer to the tip tapered foils, like rudders, flippers, wings etc. can smooth the lift coefficient curves by maintaining the lift. The performance regarding the lift-to-drag ratio can be enhanced as well as a delay in stall being observed.

In order to explore the further potential of the application of this concept in the field of tidal turbines, Newcastle University launched a series of numerical and experimental investigations to apply leading-edge tubercles to horizontal axis tidal turbine (HATT) blades. Within this framework, a tapered end 3D hydrofoil was designed based on an existing HATT blade with the same chord length distribution but with a constant pitch angle 
to separately analyze the load characteristics of the hydrofoil with and without the tubercles. The designed hydrofoil was then tested in the ECT to measure the lift and drag characteristics. To complement the experimental study and achieve more detailed flow field information and also to validate the experiments from a numerical perspective, a computational fluid dynamics (CFD) simulation study has been conducted using Star-CCM+.

In the remainder of the paper, the tested hydrofoil design and manufacturing process are first introduced together with the experimental results from the Emerson Cavitation Tunnel (ECT). The methodology of the CFD simulation is then described. A mesh convergence study and a turbulence model validation study have been performed and calibrated with the experimental results. The experimental and numerical results are then correlated to allow a more detailed study of the flow field such that a more thorough understanding is achieved based on the study in this paper.

\section{HYDROFOIL DESIGN AND TEST}

\section{Hydrofoil Design and Manufacture}

The hydrofoil was designed based on an existing model tidal turbine blade which had an S814 profile section from the NREL series, as shown in Figure 1. Wang et al [14] conducted an experimental investigation into the efficiency, slipstream wash, cavitation and noise characteristics of this turbine model. The scaled turbine model is shown in Figure 2 as mounted on the open water dynamometer of the ECT.

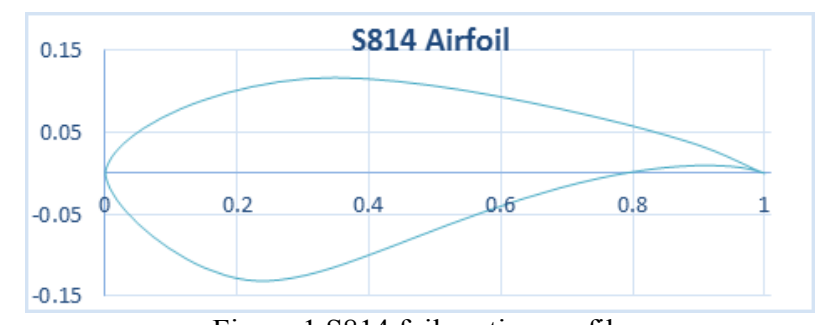

Figure 1 S814 foil section profile

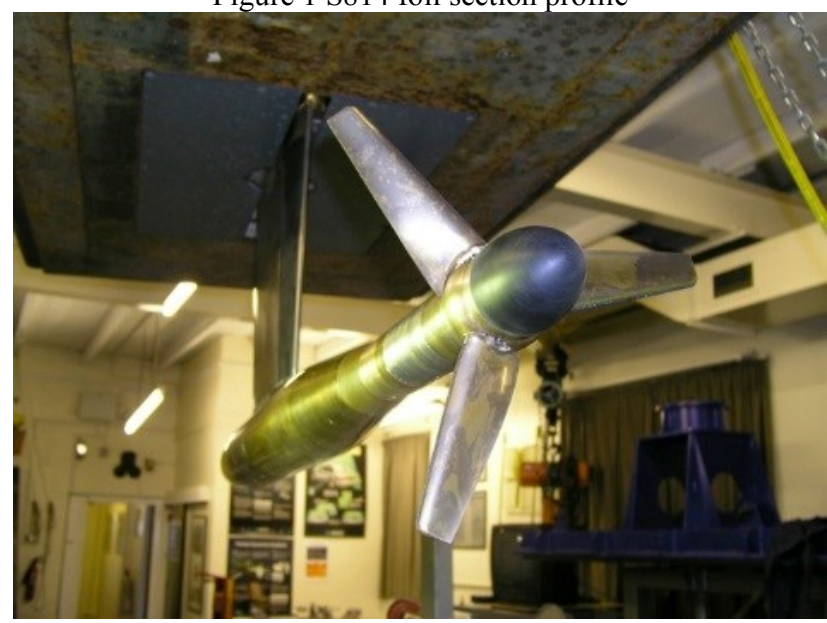

Figure 2 Scaled tidal turbine model mounted on a dynamometer
For the model tests a 3D foil based on the blade of the tidal turbine, which had the same chord length distribution as the model tidal turbine blade but with a constant pitch angle, was specified. Based on the limitations for the testing section of the ECT, a 560mm long 3D foil was designed and sectional main particulars of the foil are shown in Table 1. According to the optimisation study [15], the foil with the tubercles would display better performance in maintaining the lift when the amplitude (A) and wavelength (W) of the tubercles were $0.1 \mathrm{C}$ (Chord) and $0.5 \mathrm{C}$, respectively. Hence approximately 8 sinusoidal tubercles were evenly distributed along the leading edge of the test foil.

Table 1 Sectional particular of test hydrofoil

\begin{tabular}{|c|c|}
\hline Span(mm) & Chord(mm) \\
\hline 0 & 225.1 \\
\hline 70 & 210.08 \\
\hline 140 & 195.06 \\
\hline 210 & 180.04 \\
\hline 280 & 165.02 \\
\hline 350 & 150 \\
\hline 420 & 134.98 \\
\hline 490 & 119.96 \\
\hline 560 & 104.94 \\
\hline
\end{tabular}

Based on the above design the 3D foil was manufactured in two separate parts and assembled. The first part was the interchangeable leading-edge part of the foil while the second part was the main body of the foil that also supported the whole foil structure. The removable leading-edge was printed in four segmented pieces from plastic powder material using a 3D printer. The main body part of the foil was milled on a CNC machine from Carbon Fiber Reinforced Plastic (CFRP) to ensure that the structure was strong enough and deformation was minimal. The foil model (in black) with the smooth leading edge (in white) and tubercle leading edge (also in white) profiles are shown in Figure 3 side by side. Using the foil model, various combinations of the leading edge profiles were tested and compared including the base foil without the tubercles to understand the influence of the tubercle arrangements on the foil performance. In this paper the experimental results of the hydrofoils with the smooth leading-edge profile, named "Ref" and with the tubercle leading-edge profile, named "Sin8" were used to calibrate the CFD simulations.

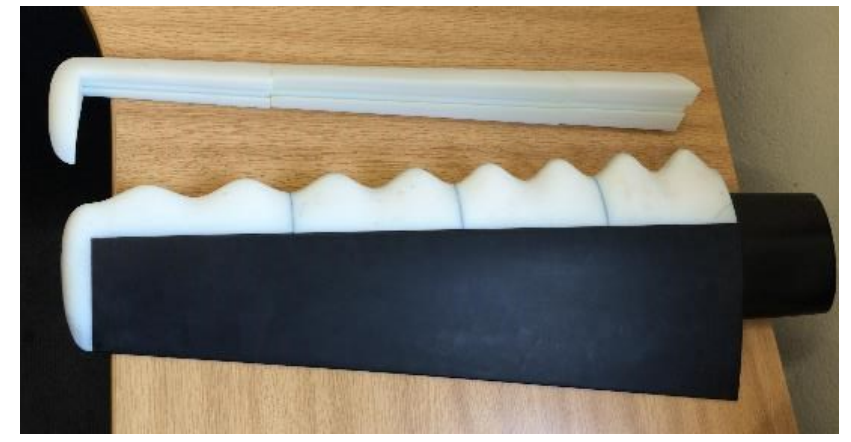

Figure 3 Tested 3D foil model 


\section{Experimental Setup}

The experiments were conducted in the Emerson Cavitation Tunnel (ECT) at Newcastle University, as shown in Figure 4. The tunnel is a medium size propeller cavitation tunnel with a measuring section of $1219 \mathrm{~mm} \times 806 \mathrm{~mm}$ (width $\times$ height). The speed of the tunnel water varies between 0.5 to $8 \mathrm{~m} / \mathrm{s}$. Full details of the ECT can be found in [16].

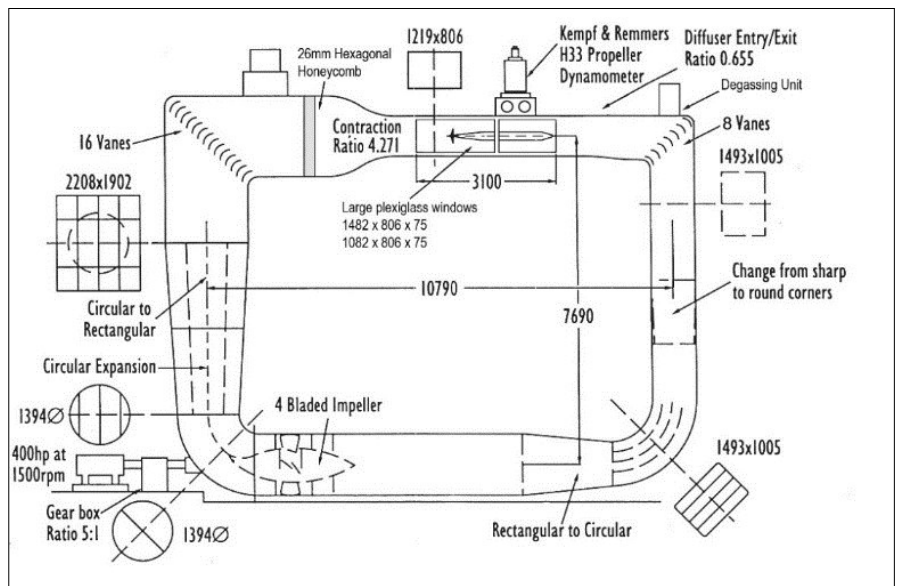

Figure 4 Emerson Cavitation Tunnel [15]

Over the operating range of the tip speed ratios (TSRs), the angle of attack (AOA) for the foil was varied from $0^{\circ}$ to $40^{\circ}$ during the tests while the inflow velocities were selected as 2, 3 and $4 \mathrm{~m} / \mathrm{s}$. Based on this inflow velocity range and the $150 \mathrm{~mm}$ foil chord length at 0.7 radius, the reference Reynolds number ranged from 0.3 to 0.6 million. This was the same range as for the turbine model that was used by Wang et al [14] in the experiments.

The lift and drag performance of the foil was the primary interest for the tests as in many foil investigations. During the tests, the forces acting on the foil, which was vertically oriented in the tunnel measuring section, were measured using the 3component balance device of the tunnel. The foil was mounted to the bottom plate of the 3-component balance to transfer the forces and covered with a circular plate to make the top of the tunnel flush, as shown in Figure 5.

The measured lift and drag forces were represented by the following non-dimensional coefficients:

$$
\begin{aligned}
& C_{L}=\frac{\text { Lift }}{\frac{1}{2} \rho V^{2} A} \\
& C_{D}=\frac{\text { Drag }}{\frac{1}{2} \rho V^{2} A}
\end{aligned}
$$

Equation 1

Equation 2

Where Lift is the measured lift on the foil; Drag is the measured drag of the foil; $\rho$ is the density of the tunnel water, which was measured as $1004 \mathrm{~kg} / \mathrm{m}^{3}$ using a density meter; $V$ is the tunnel flow velocity; $A$ is the reference area of the foil which is $0.0924 \mathrm{~m}^{2}$.

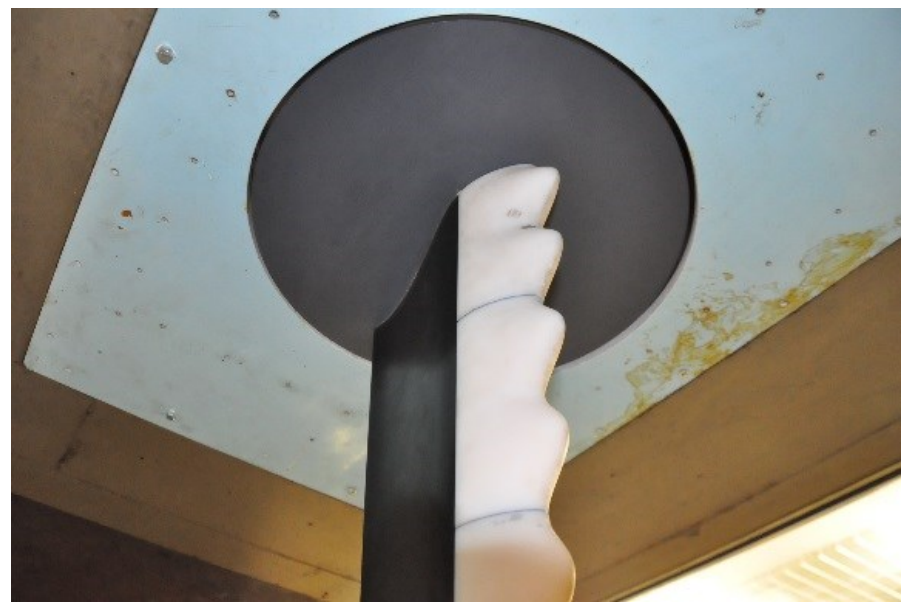

Figure 5 Foil mounted on the 3-component balance

All the measured data was gathered by a National Instruments data acquisition system and analysed instantaneously by LabVIEW. 500,000 samples were acquired at a $1 \mathrm{kHz}$ sample rate and averaged to achieve the mean value for each measurement. With this method each condition was repeated three times. The average results were then plotted and compared.

\section{Test results and Analysis}

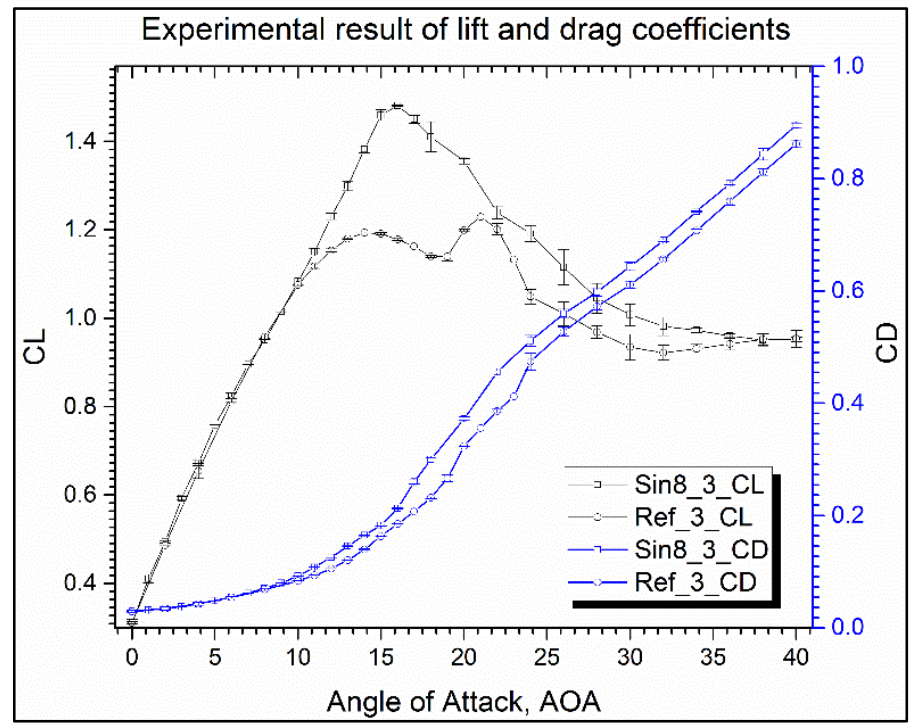

Figure 6 Experimental Lift and Drag coefficients of the reference hydrofoil (Ref) and the hydrofoil with leading-edge tubercles (Sin_8)

Figure 6 shows variation of the lift and drag coefficients over the tested angle of attack together with standard deviation. The averaged standard deviation was around $1.1 \%$ for the lift measurement and $1.4 \%$ for the drag measurement. Comparison of the measured data for the reference foil (Ref) and that of the foil (Sin_8) with the full leading-edge tubercles at $3 \mathrm{~m} / \mathrm{s}$ has been made. It clearly shows the beneficial effect of the tubercles on 
the lift coefficient. It is interesting to note that the lift coefficients of the two foils are almost identical until $9-10^{\circ}$ angle of attack after which the foil "Sin8" can maintain a linear growth until $16^{\circ}$ while the foil "Ref" cannot. However the tubercles also increase the drag coefficient after $10^{\circ}$. These test results were used to calibrate the numerical simulation in the following sections of the paper in order to explore whether the numerical simulations could accurately capture some of the physical phenomena.

\section{METHODOLOGY OF CFD SIMULATION}

The numerical simulations were conducted by using the commercial CFD software, Star-CCM+, to solve the incompressible Navier-Stokes equations with different turbulence models which are all recommended models for better prediction of the flow separations, including Shear Stress Transport (SST), Detached Eddy Simulation (DES) and Large Eddy Simulation (LES).

\section{Computational domain and boundary conditions}

The CFD simulation domain was dimensioned according to the experimental environment: the ECT testing section which is $3 \mathrm{~m}$ long, $1.2 \mathrm{~m}$ wide and $0.8 \mathrm{~m}$ high, as shown in Figure 7 . As in the experimental setup, the hydrofoil was located at $0.5 \mathrm{~m}$ downstream of the inlet and attached to the top boundary of the computational domain.

'No-slip wall' was chosen as the boundary condition of the hydrofoil, while 'free-slip wall' was used for the tunnel walls to save the mesh number on the massive prism layer of the tunnel wall. Velocity-inlet with uniform flow of $3 \mathrm{~m} / \mathrm{s}$ was used as the boundary condition for the inlet. Meanwhile the pressure-outlet condition was applied as the outlet condition.

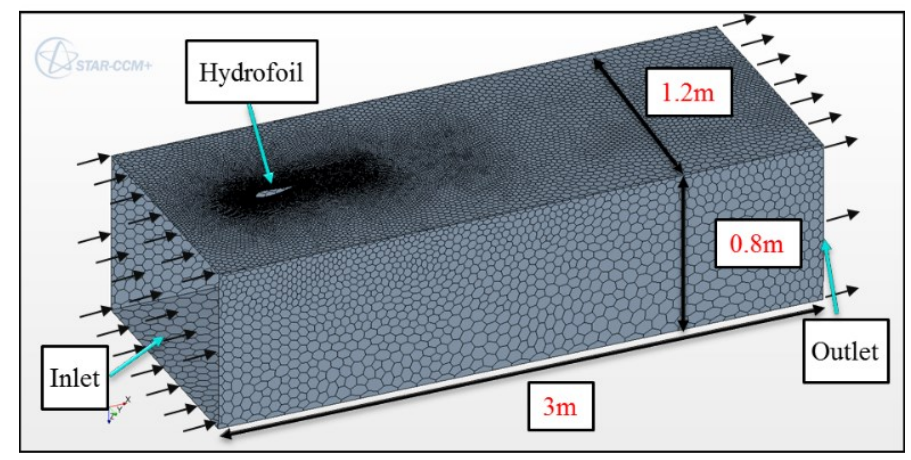

Figure 7 Computation domain and boundary conditions

\section{Mesh generation}

The mesh of this computational domain was generated by taking advantage of the automatic polyhedral mesher in Star$\mathrm{CCM}+$, which is estimated to require a four times fewer cells, half the memory and a tenth to fifth of computing time compared to tetrahedral meshes to reach solutions of the same accuracy. Volumetric mesh control was used to control the growth of the mesh. As shown in Figure 8, three blocks were used to refine the mesh near the hydrofoil: Refine 1, Refine 2 and Refine 3. The generated mesh with volumetric control can be seen in Figure 9.

To control the mesh resolution in the boundary layer, prism layer mesh was used to guarantee the near wall mesh quality. Ten layers of prism mesh were generated and the first layer thickness was set to be $1 \mathrm{E}-5 \mathrm{~m}$, as shown in Figure 10.

A mesh convergence study was performed by gradually decreasing the base size of the mesh so that the resolution of mesh was improved until it reached sufficient accuracy to match the experimental results.
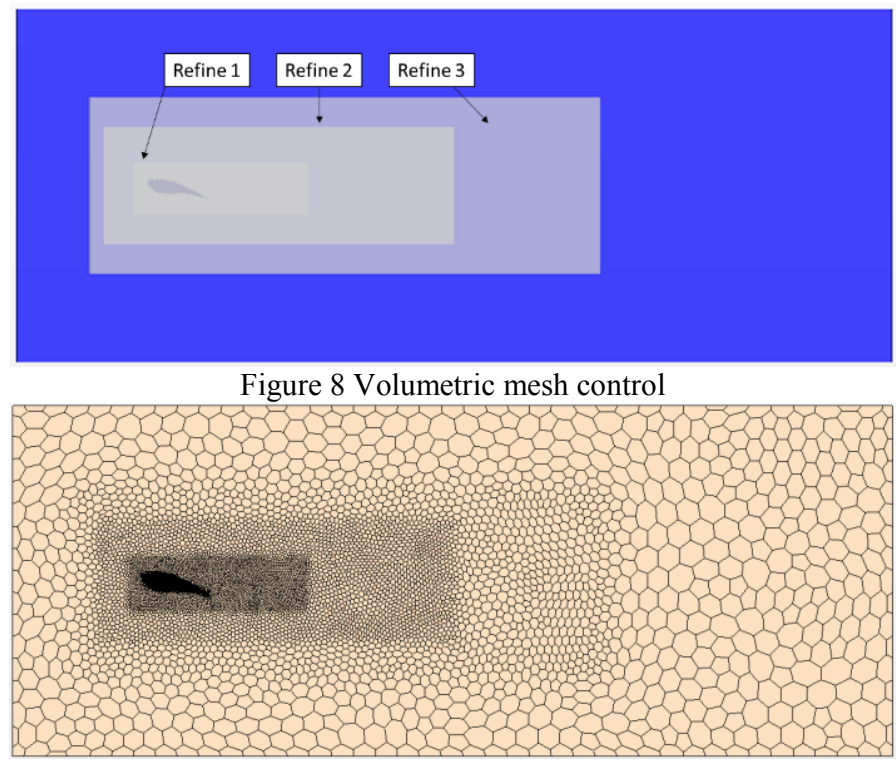

Figure 9 Generated mesh with volumetric control

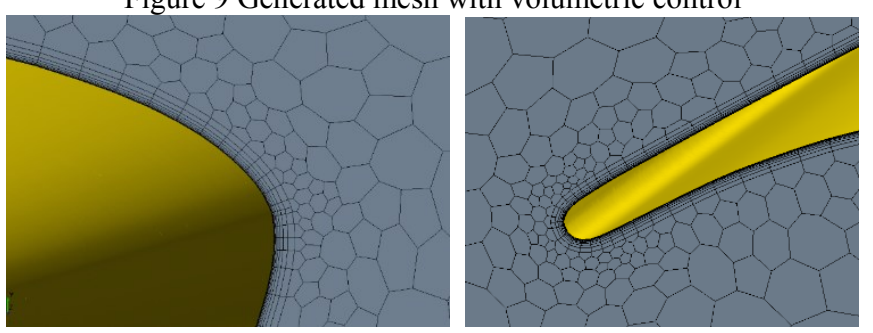

Figure 10 Prism layer mesh

\section{Turbulence model selection}

Based on the experimental study, the performance difference of different leading-edge profiles was observed in the stall condition: while the reference hydrofoil was suffering from flow separation and hence losing its lift; the one with leadingedge tubercles could delay the stall and maintain the lift. Therefore three recommended turbulence models for flow separation were used and validated with the experimental result, namely Shear Stress Transport (SST), Detached Eddy Simulation (DES) and Large Eddy Simulation (LES).

Single phase incompressible flow was modelled in this paper. However the development of tip cavitation can also be found during the experimental study which might pose an effect on the results. In the future multiphase flow with cavitation 
model would be developed. Implicit unsteady analysis was used to model the flow separation in DES and LES simulations with $0.001 \mathrm{~s}$ as the time step, while a time independent steady analysis was used in the SST turbulence model.

\section{RESULTS AND DISCUSSIONS}

\section{Convergence and validation study}

During the simulation the $C_{L}$ and $C_{D}$ coefficients were monitored to check the convergence of the simulation. One example of the convergence histogram against the iteration steps is shown in Figure 11, which shows a well converged simulation.

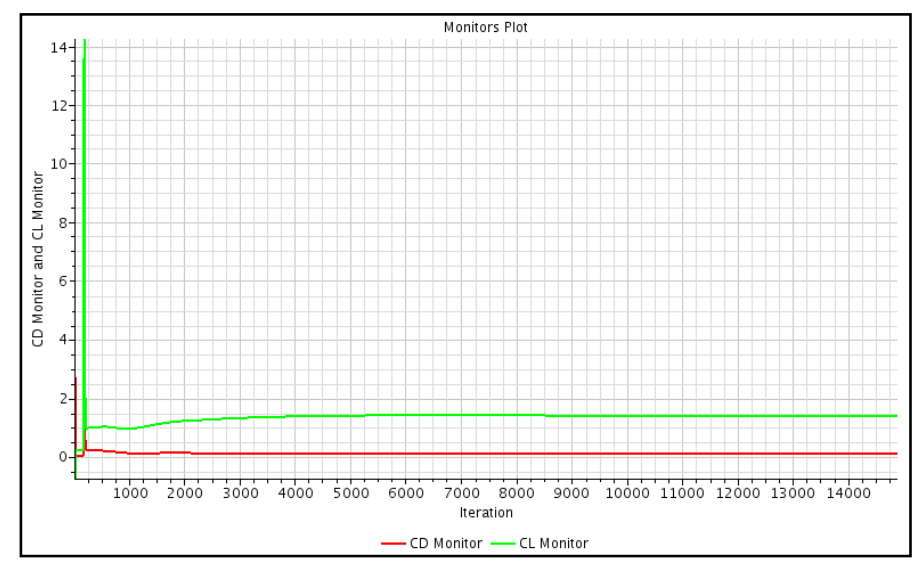

Figure 11 Example of convergence histogram of $C_{L}$ and $C_{D}$

The mesh sensitivity was conducted based on the reference hydrofoil via using 4 different mesh distributions by gradually increasing the number of element from 2 million to 21 million cells. A turbulence model verification study was also conducted by combining the mesh study with the three different turbulence models: SST, DES and LES. Four different AOAs were simulated. According to the simulated lift coefficient shown in Figure 12, DES model with a 21 million cell mesh shows the best agreement with the experimental data, CL, even at the stall conditions, which is very important for this simulation.

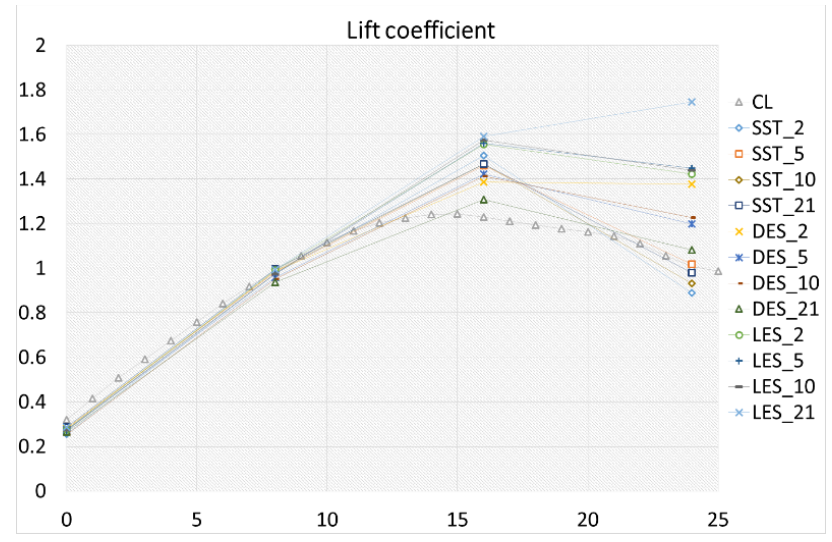

Figure 12 Mesh sensitivity and turbulence model verification

\section{Force characteristics}

Based on the experimental study, the following analysis was focused on the most significant condition when the AOA is equal to $16^{\circ}$. The result is presented in Table 2 . It can be seen that the numerical model can distinguish the difference between these two different leading-edge designs and shows a close agreement with the experimental data in lift coefficients but around $10 \%$ error in drag coefficients. This discrepancy on drag coefficient might be because of the uncertainty of the skin friction introduced by the surface characteristics of the tested model since the 3D printed leading-edge tubercles have a relatively rough surface. However because of the nature of this comparison study, the numerical results of two hydrofoils show the same level of disparity.

Table 2 Experimental and numerical results of the hydrofoil with and without tubercles at $\mathrm{AOA}==16^{\circ}$

\begin{tabular}{|c|c|c|}
\hline \multicolumn{3}{|c|}{ without tubercles at $\mathrm{AOA}==16^{\circ}$} \\
\hline Ref_EXP & 1.22902 & 0.17447 \\
\hline Ref_DES & 1.25 & 0.1515 \\
\hline Sin8_EXP & 1.48132 & 0.21297 \\
\hline Sin8_DES & 1.45 & 0.1900 \\
\hline
\end{tabular}

\section{Flow patterns}

The flow separation patterns at $\mathrm{AOA}=16^{\circ}$ for the reference hydrofoil and the one with tubercles are shown in Figure 13 using iso-surface presentation for the axial velocity $(\mathrm{Vx})=0 \mathrm{~m} / \mathrm{s}$. It can be clearly seen that the iso-surface of the reference hydrofoil was continuous, forming into a huge cloud along trailing edge while the iso-surface of the hydrofoil with tubercles was separated by the tubercles. The volume was also much smaller than for the reference case even though in the trough area the flow separation was slightly earlier in the leading-edge region than that of the reference hydrofoil.

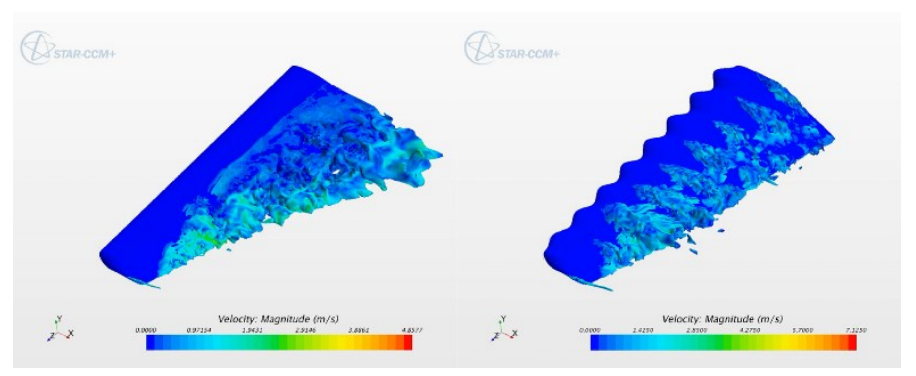

Figure 13 Flow separation pattern (Iso-surface: $\mathrm{Vx}=0 \mathrm{~m} / \mathrm{s} ; \mathrm{AOA}=16^{\circ}$ )

\section{Vorticity distribution}

Following the observation of the flow separation pattern, a section cut was extracted from the flow field to see the tip vortices and the vorticity distribution along the span as shown in 
Figure 14. A strong tip vortex can be generated by both hydrofoils. While comparing the vorticity distributions of the two hydrofoils along the span direction, one can observe a low vorticity flow activity with the reference foil inside the flow separation line with an increasing slope. However the foil with tubercles shows a highly energized flow with high vorticity, which is also more attached to the surface, compared to the reference foil.

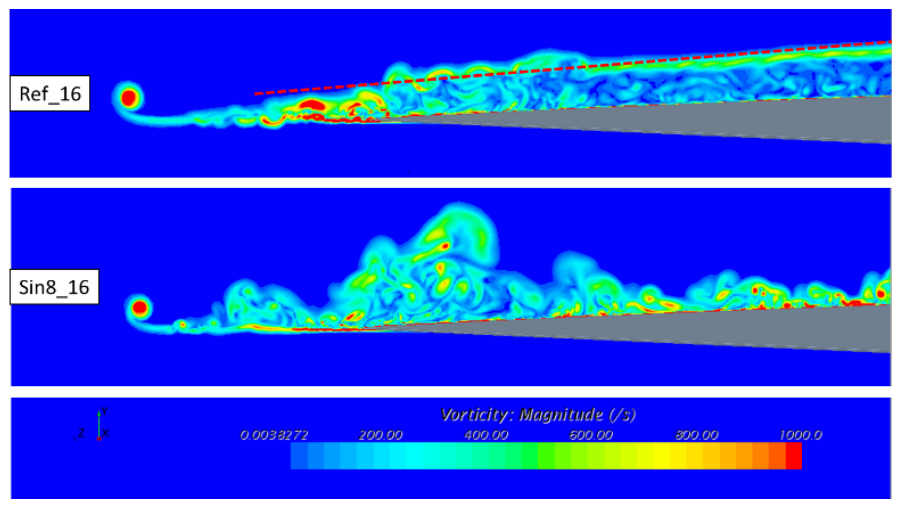

Figure 14 Vorticity of tip vortex and flow separation at section $\mathrm{X}=0.2 \mathrm{~m}$

By extracting the vorticity distributions on the hydrofoils, in Figure 15, the flow separation patterns can be clearly compared. One can notice that the flow on the hydrofoil with tubercles is much more disturbed and energetic. Furthermore, Figure 16 shows the comparative vorticity [i] distributions in the perpendicular plane to the incoming velocity on the suction sides of both foils. In this figure one can see that a series of pairs of contra rotating vortices that have one positive and the other negative vorticity [i] were generated alongside the tubercles.

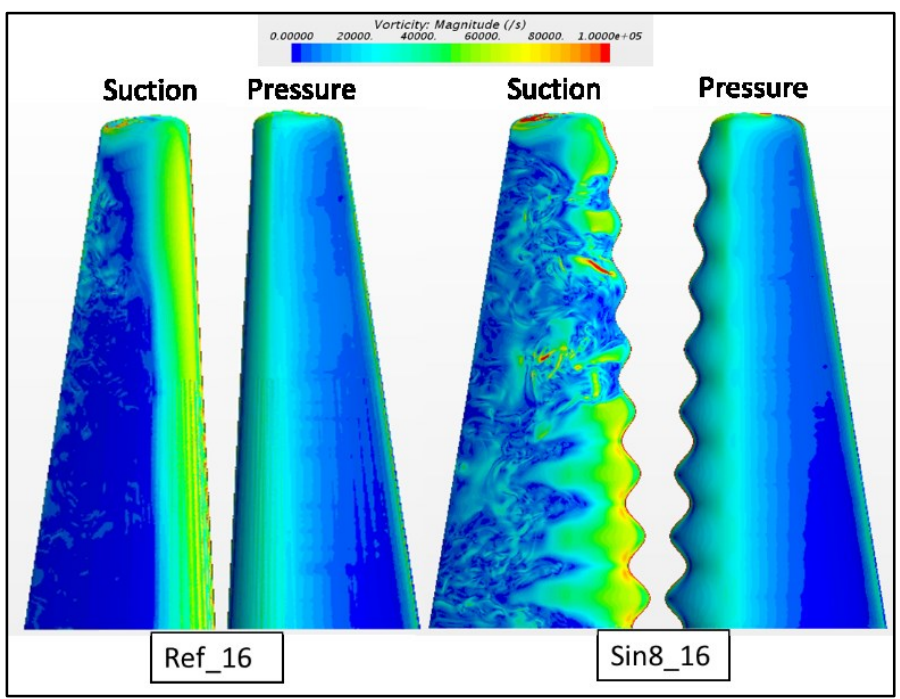

Figure 15 Vorticity magnitude distribution along the hydrofoil

According to the research which has been conducted both in the numerical and experimental fields on tubercles $[1,6,9,13$,
17-20], the leading-edge tubercles can improve the lift-to-drag ratio for tapered $3 \mathrm{D}$ foils while they can only avoid the sudden stall phenomenon for a 2D infinite foil with a penalty that would increase the drag and reduce the maximum lift coefficient. By scrutinizing the details of the vorticity [i] distribution at the tip of the hydrofoil, the secret of why the leading-edge tubercles can always perform better for a $3 \mathrm{D}$ foil than for a $2 \mathrm{D}$ foil is revealed. The contra rotating vortices generated by the tubercles interact with the tip vortex, stopping it from spreading on the surface of the hydrofoil. As shown in Figure 17, a vortex generated by the tip tubercle which shows a positive vorticity [i] from the span position 0.525 to 0.56 cancelled the strong negative tip vortex. Because of this, the detrimental effect of the tip vortex that is generally blamed for the performance loss is greatly weakened.

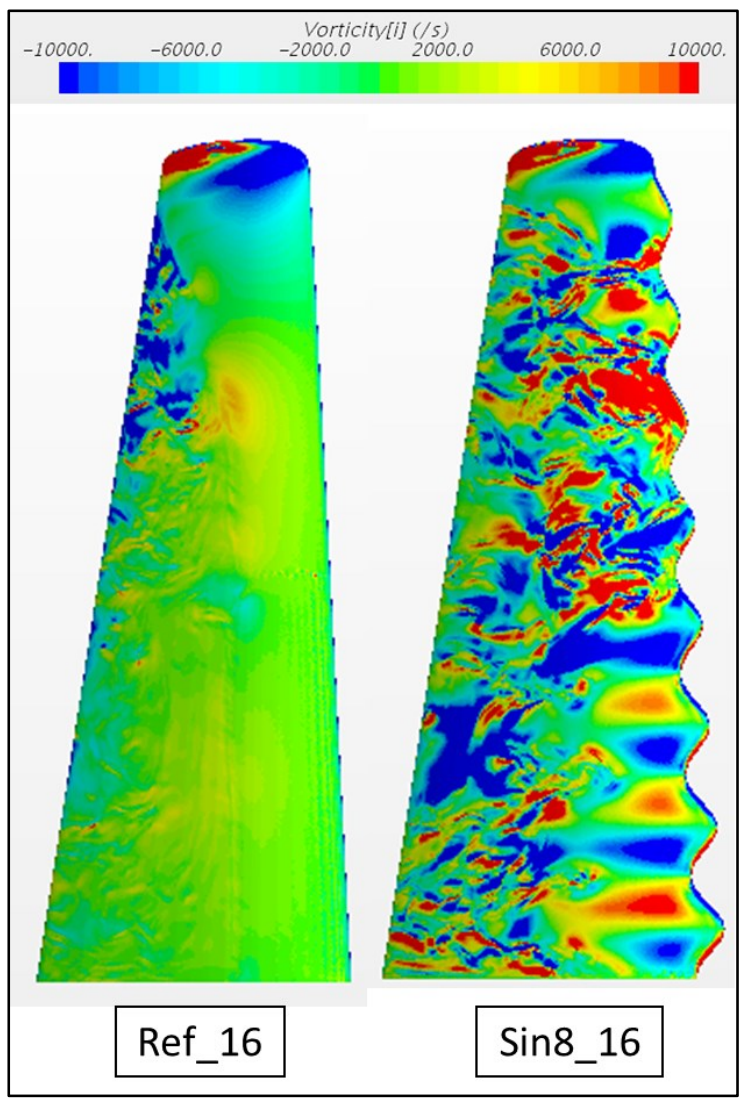

Figure 16 Vorticity [i] distribution along the hydrofoil on the suction side

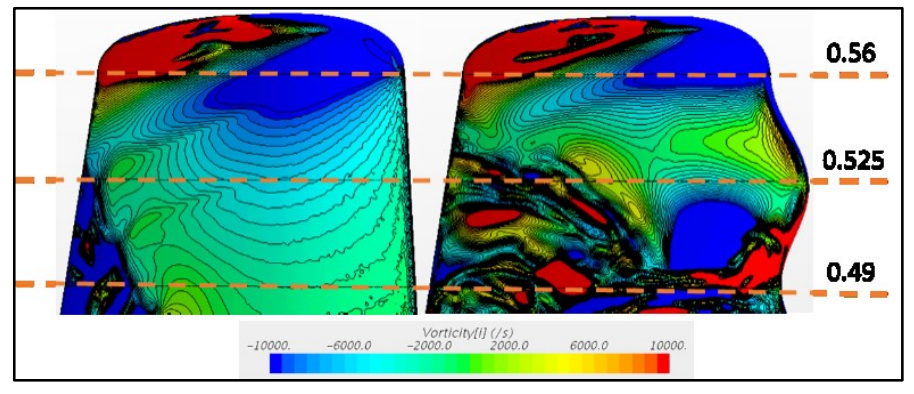

Figure 17 Details of vorticity [i] distribution at the tip 


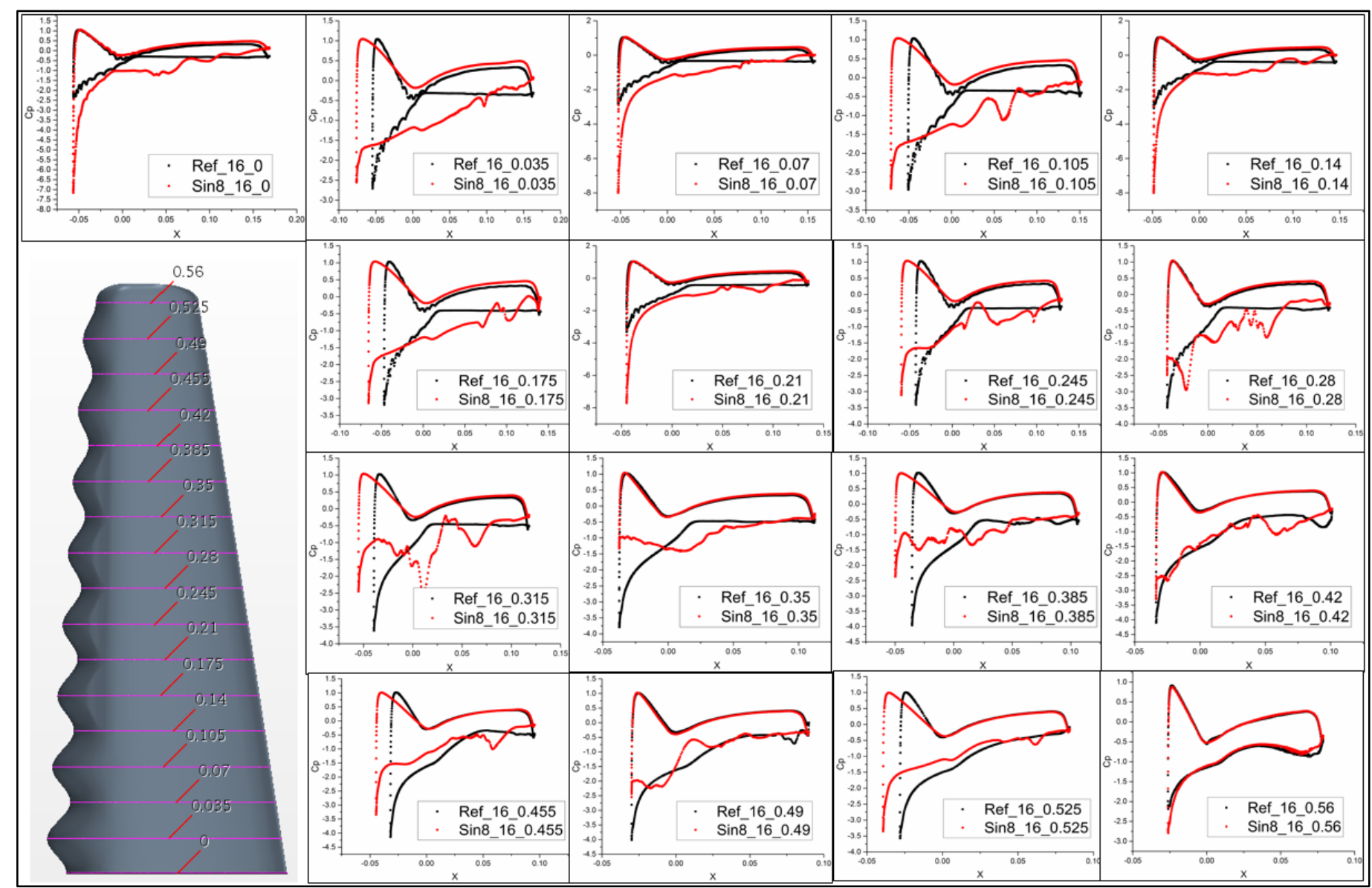

Figure 18 Comparison of pressure coefficient distributions in trough and crest area $\left(\mathrm{AOA}=16^{\circ}\right)$

\section{Pressure distributions}

The pressure coefficient $(\mathrm{Cp})$ was extracted from the simulation to investigate the pressure distribution on the surface of the hydrofoils. The definition of $\mathrm{Cp}$ is given by Equation 3 .

$$
C_{P}=\frac{\text { Pressure }}{\frac{1}{2} \rho V^{2}}
$$

Equation 3

Figure 18 shows the calculated pressure coefficients at the same sections, which were taken along the span of hydrofoils at the consecutive locations of troughs and crests. In Figure 18, the red dots represent the hydrofoil with tubercles (Sin8) while the black ones represent for the reference hydrofoil (Ref).

It is well known that the integration of the pressure distribution curve along the pressure side and the suction side produces the lift of the foils. As it can be seen, apart from the tip region that generally the red dots cover a larger area than do the black dots. For the position in the trough of the tubercles, a better suction effect of negative $\mathrm{Cp}$ is shown while similar pressure distribution on the pressure side can be maintained. This is because of the more attached flow as shown in Figure 13. For the position of the crest region, first the chord length of Sin8 is $10 \%$ longer than the reference one. At the same time a better suction effect can also be observed in mid-chord region. However, this feature is gradually reduced close to the tip because of the influence caused by the tip vortex.

\section{CONCLUSIONS}

In this study the fundamental effects of leading edge tubercles on the hydrodynamic characteristics of a representative 3D hydrofoil, which is based on a tidal turbine blade, have been investigated using experimental and CFD based methods. Based on the investigation the following conclusions can be drawn:

1. Comparative model tests in a cavitation tunnel for the 3D foil with and without leading-edge tubercles confirmed the significant benefits of the tubercles on the lift of the foils despite an increase in their drag characteristics.

2. A CFD simulation has been conducted to complement the experimental study for more detailed flow analysis. The DES model with a 21 million cell mesh showed a 
close agreement with the experimental data for the lift coefficient. The numerical simulation also confirmed that the most beneficial effect caused by the leadingedge tubercles was at $16^{\circ}$ angle of attack.

3. According to post analysis of the flow pattern in terms of vorticity distribution and pressure distribution, the flow around the hydrofoil with tubercles is much more energetic. This is caused by a series of pairs of contra rotating vortices generated by the tubercles along the foil span. These vortices help the flow to be more attached to the surface at the expense of more energy being consumed which leads to the drag increase.

4. However, the detrimental effect of the tip vortex is greatly reduced, because of the favourable cancellation effect of the contra-rotating vortices with the tip vortex. This reveals the major difference of the effect of tubercles on 3D foils and 2D foils.

\section{ACKNOWLEDGMENTS}

This research is funded by the School of Marine Science and Technology, Newcastle University and the China Scholarship Council. Hence the financial support obtained from both establishments is gratefully acknowledged. The authors would also like to thank all the team members in the Emerson Cavitation Tunnel for their help in testing and sharing their knowledge.

\section{REFERENCES}

[1] Fish, F. E., Weber, P. W., Murray, M. M., and Howle, L. E., 2011, "The tubercles on humpback whales' flippers: application of bio-inspired technology," Integrative and comparative biology, 51(1), pp. 203-213.

[2] Frank E. Fish, and Battle, J. M., 1996, "Hydrodynamic design of the humpback whale flipper," Journal of Morphology.

[3] K. L. Hansen, R. M. Kelso, and Dally, B. B., 2009, "The effect of leading edge tubercle geometry on the performance of different airfoils."

[4] Yoon, H. S., Hung, P. A., Jung, J. H., and Kim, M. C., 2011, "Effect of the wavy leading edge on hydrodynamic characteristics for flow around low aspect ratio wing," Computers \& Fluids, 49(1), pp. 276-289.

[5] Johari, H., Henoch, C., Custodio, D., and Levshin, A., 2007, "Effects of leading-edge protuberances on airfoil performance," Aiaa J, 45(11), pp. 2634-2642.

[6] Miklosovic, D. S., Murray, M. M., and Howle, L. E., 2007, "Experimental evaluation of sinusoidal leading edges," J Aircraft, 44(4), pp. 1404-1408.

[7] Stanway, M. J., 2008, "Hydrodynamic effects of leading-edge tubercles on control surfaces and in flapping foil propulsion," Massachusetts Institute of Technology.

[8] Weber, P. W., Howle, L. E., and Murray, M. M., 2010, "Lift, drag, and cavitation onset on rudders with leading-edge tubercles," Mar Technol Sname N, 47(1), pp. 27-36.

[9] van Nierop, E., Alben, S., and Brenner, M., 2008, "How bumps on whale flippers delay stall: An aerodynamic model," Physical Review Letters, 100(5).
[10] Corsini, A., Delibra, G., and Sheard, A. G., 2013, "On the role of leading-edge bumps in the control of stall onset in axial fan blades," J Fluid Eng-T Asme, 135(8), pp. 081104-081104.

[11] Hansen, K. L., Kelso, R. M., and Dally, B. B., 2011, "Performance variations of leading-edge tubercles for distinct airfoil profiles," Aiaa J, 49(1), pp. 185-194.

[12] Mark W. Lohry, D. C. a. L. M., 2012, "Characterization and Design of Tubercle Leading-Edge Wings," Seventh International Conference on Computational Fluid Dynamics (ICCFD7)Big Island, Hawaii.

[13] Chen, J. H., Li, S. S., and Nguyen, V. T., "The effect of leading edge protuberances on the performance of small aspect ratio foils."

[14] Wang, D., Atlar, M., and Sampson, R., 2007, "An experimental investigation on cavitation, noise, and slipstream characteristics of ocean stream turbines," Proceedings of the Institution of Mechanical Engineers, Part A: Journal of Power and Energy, 221(2), pp. 219-231.

[15] Shi, W., Atlar, M., Norman, R., and Seo, K.-c., 2014, "CFD investigations on leading-edge tubercles as applied on a tidal turbine blade," GRAND RENEWABLE ENERGY 2014Tokyo, Japan.

[16] Atlar, M., "Recent upgrading of marine testing facilities at Newcastle University," Proc. AMT'11, the second international conference on advanced model measurement technology for the EU maritime industry, pp. 4-6.

[17] Rostamzadeh, N., Hansen, K. L., Kelso, R. M., and Dally, B. B., 2014, "The formation mechanism and impact of streamwise vortices on NACA 0021 airfoil's performance with undulating leading edge modification," Phys Fluids, 26(10), pp. $1-22$.

[18] Rostamzadeh, N., Kelso, R. M., Dally, B. B., and Hansen, K. L., 2013, "The effect of undulating leading-edge modifications on NACA 0021 airfoil characteristics," Phys Fluids, 25(11).

[19] Wei, Z., New, T. H., and Cui, Y. D., 2015, "An experimental study on flow separation control of hydrofoils with leading-edge tubercles at low Reynolds number," Ocean Engineering, 108, pp. 336-349.

[20] Miklosovic, D. S., Murray, M. M., Howle, L. E., and Fish, F. E., 2004, "Leading-edge tubercles delay stall on humpback whale (Megaptera novaeangliae) flippers," Phys Fluids, 16(5), pp. L39-L42. 